\title{
Juvenile Ossifying Fibroma of the Maxilla: A Case Report
}

\author{
*MS Kamal ${ }^{1}$, MAA Bahar ${ }^{2}$, MA Latif ${ }^{3}$, MMIU Khan ${ }^{4}$ \\ ${ }^{* 1}$ Dr. Mohammad Shah Kamal, Junior consultant (ENT), Shaheed Shamsuddin Ahmed Hospital, Sylhet, Bangladesh \\ ${ }^{2}$ Dr. Mohammad Ashik Anwar Bahar, Junior consultant (surgery), Shaheed Shamsuddin Ahmed Hospital, Sylhet, Bangladesh \\ ${ }^{3}$ Dr. MA Latif, Junior consultant (Anaesthesia), Shaheed Shamsuddin Ahmed Hospital, Sylhet, Bangladesh \\ ${ }^{4}$ Dr. Mehdi Mohammad Imtiaz Uddin Khan, Dental surgeon, Shaheed Shamsuddin Ahmed Hospital, Sylhet, Bangladesh \\ *Corresponding Author
}

\begin{abstract}
Juvenile ossifying fibroma (JOF) is a rare fibro-osseous neoplasm that arises within the craniofacial bones in individuals under 15 years of age. It affects both males and females equally. It has the potential for excessive growth, bone destruction, and recurrence. It is more aggressive than ossifying fibroma. Recurrence rate ranges from $30 \%$ to $58 \%$.We report a case of 11-year-old male child presented with a painless, progressive swelling of the right face for 8 months. CT scan demonstrated a well-defined, mixed-density mass filling the right maxillary sinus. Under general anaesthesia, surgical excision of the tumour was performed. Histopathologically, excised specimen was identical with fibro-osseous lesion. Juvenile ossifying fibroma (JOF) is aggressive in nature and recurrence rate is high, so early detection and complete surgical excision is essential.
\end{abstract}

Key words: Juvenile ossifying fibroma, Ossifying fibroma, Fibrous dysplasia, Fibro-osseous lesion.

\section{Introduction}

Juvenile ossifying fibroma (JOF) is a rare fibro osseous neoplasm that arises within the craniofacial bones in individuals under 15 years of age ${ }^{1}$. It is usually asymptomatic, achieving a large size and exhibiting aggressive behavior ${ }^{2}$. It affects both males and females equally. It has the potential for excessive growth, bone destruction, and recurrence. It is more aggressive than ossifying fibroma. Recurrence rate ranges from $30 \%$ to $58 \%^{3,4}$.

Radiological changes are of a discrete mass with a distinct boundary and thinning of the cortical bone, resulting in an egg-shell appearance ${ }^{3}$. The lesion is non-encapsulated but well demarcated from surrounding bone. Microscopically, juvenile ossifying fibroma is highly cellular and contains trabeculae or spheroids of new bones. Juvenile ossifying fibroma has also been termed Juvenile aggressive ossifying fibroma, Juvenile active ossifying fibroma 4 .

\section{Case report}

An 11-year-old male child presented in February 2012 wit, h a painless, progressive swelling of the right face for 8 months. He had no noteworthy family history or past history.
Clinical examination revealed a swelling with normal appearing skin on the right side of the face localized to the maxilla. No cervical lymphadenopathy was noted and the mouth opening was normal. Oral examination revealed gross expansion of the right maxillary alveolar process and bulging of the hard palate. There was no tenderness on palpation.

CT scan demonstrated a well-defined, mixeddensity mass filling the right maxillary sinus. The mass causing destruction of adjacent upper jaw, inferior orbital plate, medial and lateral wall of right maxilla. The clinical and radiological features were in favour of a fibroosseous lesion. So a diagnosis of right maxillary ossifying fibroma was made.

Under general anaesthesia, a maxillary right buccal vestibular incision was made and surgical excision of the tumour was performed. The tumour (about $5 \times 5 \mathrm{~cm}$ ) was removed completely; a clear demarcation was evident between the tumour and healthy bone. Bony defects were filled with absorbable gelatin sponge. Histopathologically, excised specimen was identical with fibro-osseous lesion. 


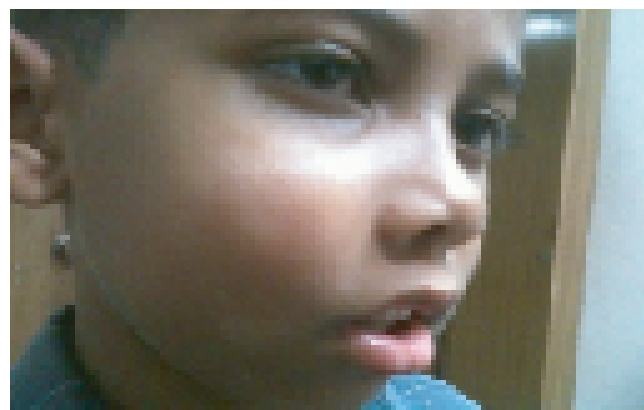

Figure 1: Swelling of right side of the face

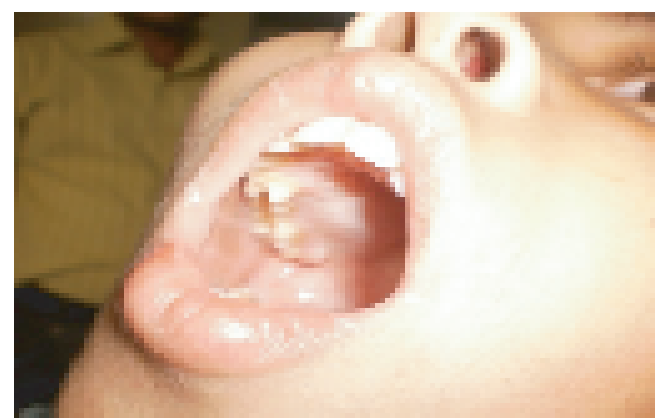

Figure 2: Intraoral view

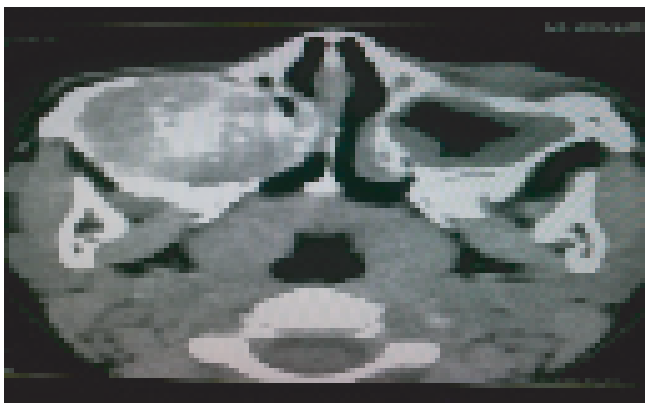

Figure 3: CT scan axial view showing bone destruction of medial wall of right maxillary sinus

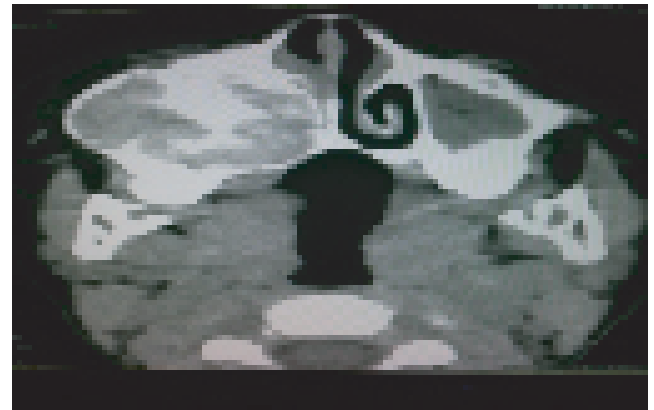

Figure 4: CT scan axial view showing mixed density lesion in the sinus.

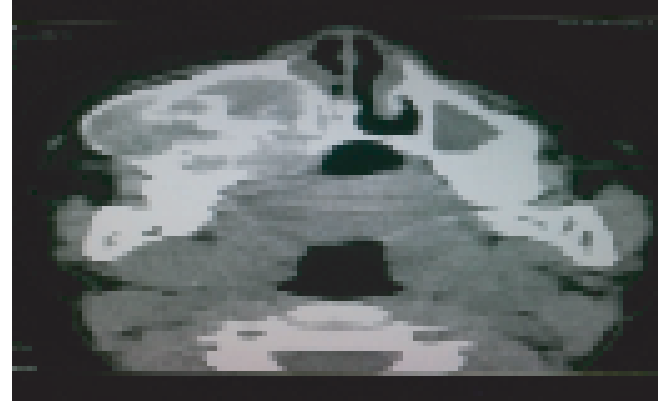

Figure 5: CT scan axial view showing destruction of maxillary sinus wall (post)

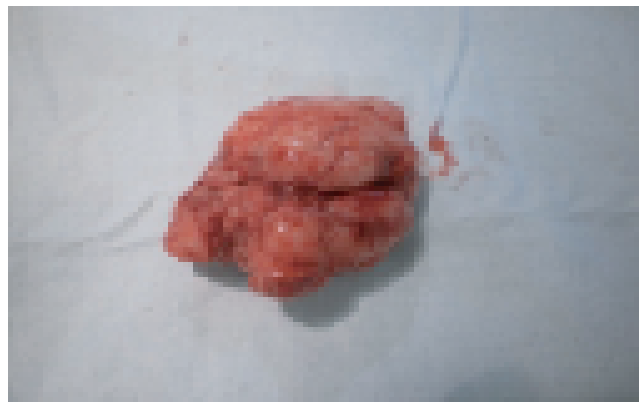

Figure 6: The mass.

\section{Discussion}

A fibro-osseous lesion is one in which bone is replaced by cellular fibrous tissue, which gradually matures with the formation of woven bone, lamellar bone or very dense amorphous mineralisation. The group includes disorders ranging from fibrous dysplasia to the circumscribed lesions of ossifying fibroma and the cemental dysplasia5. Johnson et al reviewed 3000 fibro-osseous lesions and found that majority of tumours were located in facial bones, among which approximately $90 \%$ originated from paranasal sinuses. Single or multiple sinuses may be involved. When jaw is involved, maxilla is more frequently than mandible6.

Ossifying fibroma is a benign neoplasm of bone that has the potential for excessive growth, bone destruction, and recurrence. It is a slowgrowing, asymptomatic, and expansile lesion4. Ossifying fibroma arises exclusively in the jaw, facial bones and skull5. Juvenile ossifying fibroma most commonly involves the paranasal sinuses and periorbital bones, where it may cause exopthalmos, proptosis, sinusitis, and nasal symptoms. This rare tumour behaves in a more aggressive fashion than does ossifying 
fibroma ${ }^{4}$. Juvenile ossifying fibroma affects both males and females equally ${ }^{7}$.

Radiological changes are of a discrete mass with a distinct boundary and thinning of the cortical bone, resulting in an egg-shell appearance. In most instances, the mass is surrounded by smooth, well-defined cortical bone which differentiates it from fibrous dysplasia in which 'blending' into the surrounding bone is universal. Its behaviour is that of a benign tumour and thus it continues to grow after skeletal maturation ${ }^{3}$.

Histologically, Ossifying fibroma is composed of fibrous connective tissue with welldifferentiated spindled fibroblast. Cellularity is uniform but may vary from one lesion to the next. Collagen fibres are arranged haphazardly. Bony spheroids, trabeculae, or islands are evently distributed throughout the fibrous stroma. Bone is immature and often surrounded by osteoblast. Osteoclasts are infrequently seen 4 .

Review of the literature shows that it is difficult to establish definite diagnostic criteria for JOF. The main criteria are- patient under 15 years of age, location of the tumour, radiologic pattern, aggressive clinical course, cellular(benign) stroma, trabecular or spherical bone, and tendency to recur ${ }^{1,4}$. In reviews published by Hamner et al. 8 and Slootweg et al. 9 the mean age of onset was 11.5 and 11.8 years of age respectively.

Differential diagnoses are Fibrous dysplasia, osteoblastoma, focal cement-osseous dysplasia, and focal osteomyelitis. Distinguishing between ossifying fibroma and fibrous dysplasia is the primary diagnostic challenge. Both lesions may exhibit similar clinical, radiographic, and microscopic features. The most helpful feature in distinguishing the two is the wellcircumscribed radiographic appearance of ossifying fibroma and the ease with which it can be separated from normal bone. Osteoblastoma is evident in a slight younger age group and is often characterized by pain. In addition osseous trabeculae in these lesions are rimmed by abundant plump osteoblasts, and a central nidus may be evident. Periapical cement-osseous dysplasia in posterior teeth may appear radiographically similar and require biopsy to separate it from ossifying fibroma. Focal osteomyelitis is associated with a source of inflammation and is possibly accompanied by pain and swelling 4 .
The clinical management and prognosis of JOF is somewhat uncertain. Small lesion can be treated conservatively by curettage or enucleation. An open surgical approach, such as transfacial, is ideal for resecting large and irregular shaped tumours that infiltrate sinuses and fronto-nasal bones ${ }^{10}$. Recurrence rate ranges from $30 \%$ observed by Johson et al. 6 to $58 \%$ reported by Makek ${ }^{11}$.

\section{Conclusion}

Early detection and complete surgical excision followed by long term follow-up are important in the management of Juvenile ossifying fibroma due to its aggressive nature and high recurrence rate.

\section{References}

1. Sun G, Chen X, Tang E, et al. Juvenile ossifying fibroma of the maxilla. Int.J.Oral and Maxillofac. Sur 2007; 36: 82-85.

2. EL-Mofty S. Psammomatoid and trabecular juvenile ossifying fibroma of the craniofacial skeleton: two distinct clinicopathologic entities. Oral Sur Oral Med Oral Pathol Oral Radiol Endod 2002; 93: 296-304.

3. Milford CA, Kerr RS. Anterior and anterolateral skull base and craniofacial surgery. In: Gleeson $\mathrm{M}$, et al.(editors). Scott-Brown's Otorhinolaryngology, Head and Neck Surgery, seventh edition, 3; Hodder Arnold 2008; 4124.

4. Jeffery C, Stewart B. Benign Nonodontogenic Tumors. In: Regezi JA, Sciubba JJ, Jordan RCK (editors).Oral Pathology Clinical pathologic Corelations, fourth edition, Saunders 2003, 289-94.

5. Cawson RA, Odell EW. (editors). Cawson's essentials of oral pathology and oral medicine, eighth edition. Churchil Livingstone Elsevier 2008; 146-83.

6. Johnson LC, Yousefi M, Vinh TN, et al. Juvenile active ossifying fibroma: its nature, dynamics and origin. Acta Otolaryngol Supple 1991; 488:1-40.

7. Waldron CA. Fibro-osseous lesions of the jaw. J Oral Maxillofac Sur 1993; 51: 828-35.

8. Hamner JE, Gamble JW, Gallegos GJ. Odontogenic fibroma. Report of two cases. Oral Sur Oral Med Oral Pathol 1996; 21: 113-19.

9. Slootweg PJ, Panders AK, Koopmans R, et al. Juvenile ossifying fibroma. An analysis of 33 cases with emphasis on histopathological aspect. J Oral Pathol Med 1994; 23: 385-388.

10. Lawton MT, Heiserman JE, Coons SW, et al. Juvenile Active ossifying fibroma: Report of four cases. J Neurosurg 1997; 86: 279-85.

11. Makek MS. So called "fibro-osseous lesions" of tumour origin. Biology confronts terminology. J Craniomaxillofac Sur 1987; 15: 154-67. 